\title{
Mechanism of optical degradation in microstructured InGaN light-emitting diodes
}

\author{
Z. L. Li, K. H. Li, and H. W. Choi ${ }^{\text {a) }}$ \\ Semiconductor Lighting and Display Laboratory, The University of Hong Kong, Hong Kong
}

(Received 18 June 2010; accepted 22 October 2010; published online 10 December 2010)

\begin{abstract}
While the enhancement of light extraction efficiency from microstructured InGaN light-emitting diodes ( $\mu$ LED) has been firmly established, there is concern over the effect of microstructuring on the device lifetimes. A study on the electrical characteristics and reliability of $\mu$ LED arrays has been carried out. Despite improved optical performance, expanded device sidewalls served to accelerate the rate of optical degradation, adversely affect the lifetimes of devices. Through current-voltage plots and noise spectrum measurements, vertical current conduction along the plasma-damaged sidewalls was identified as the key degradation mechanism. () 2010 American Institute of Physics. [doi:10.1063/1.3517829]
\end{abstract}

\section{INTRODUCTION}

The external quantum efficiency of light-emitting diodes (LEDs) is determined by two main factors: its internal quantum efficiency which is material-dependent, and light extraction efficiency which is determined by design of the device. In order to bridge the gap between the potential of LEDs and the capabilities of present-day devices, extensive efforts in optimizing material growth and device design have been made. Microstructured light-emitting diodes ( $\mu$ LED), consisting of interconnected arrays of micrometer scale lightemitting pixels, have been demonstrated as a feasible method to enhance light extraction by providing additional photon escape pathways through sidewalls of microstructures. ${ }^{1,2}$

The proven advantage of $\mu$ LEDs over large-area LEDs is its distinctively higher optical output. Photons can be extracted from the sidewalls of the multiple micrometer-scale elements, resulting in reduced re-absorption (and thus heat generation). Many studies on the effects of dimensions and geometry (microdisks, ${ }^{1}$ microrings, ${ }^{3}$ and microhexagons ${ }^{4}$ for instance) have been reported. While the benefits of enhanced light extraction are firmly established, there inevitably will be concerns over the reliability of $\mu$ LEDs as LEDs are expected to be light sources of exceptionally long lifetime. The formation of a $\mu \mathrm{LED}$ network involves dry etching through the multiquantum wells (MQWs) removing the materials between $\mu$ LED elements, exposing additional sidewalls. Since the plasma-damaged sidewall terminations are in fact twodimensional defects, the electrical characteristics and reliability of such devices are potential affected. As a matter of fact, the distinctive geometries of $\mu$ LEDs facilitate failure analysis of LED devices.

In this paper, the mechanisms of degradation in $\mu$ LEDs are reported. Interconnected $\mu$ LEDs of hexagonal and circular geometries are fabricated and characterized, and compared with conventional large area rectangular LEDs. The devices are not passivated after dry etching in order to study the true impact of microstructuring. Device optical spectrum,

${ }^{\text {a)} E l e c t r o n i c ~ m a i l: ~ h w c h o i @ h k u . h k . ~}$ together with electrical performance, including I-V characteristics, leakage current and noise spectrum are measured. The devices subsequently undergo a $1000 \mathrm{~h}$ continuous constant current stress test to determine the optical degradation rate, which is correlated with the device geometries. The effects of vertical conduction along sidewalls on device performance and reliability are discussed.

\section{EXPERIMENTAL DETAILS}

The structure of the LED wafer used in this study consists of a GaN buffer layer grown on c-plane sapphire substrate, an n-type GaN layer, InGaN/GaN quantum wells, capped with a top contact p-type GaN layer. The emission center wavelength of the wafer is $\sim 465 \mathrm{~nm}$. The LEDs were fabricated using a standard microfabrication process beginning with the deposition of an indium-tin-oxide (ITO) current-spreading layer. The active regions of different geometries were defined by photolithography and dry etched by reactive ion etching using a gas chemistry comprising $\mathrm{CHF}_{3}$ and $\mathrm{Ar}$ to expose the $\mathrm{n}$-contact regions. For $\mu$ hexagon LEDs, each emissive element is in the shape of a hexagon, with side lengths of $25 \mu \mathrm{m}$. These are in turn hexagonally packed into a $12 \times 14$ array with a separation of $4 \mu \mathrm{m}$. For $\mu$ disk LEDs, each emissive element is a disk with a diameter of $50 \mu \mathrm{m}$ and an array consists of $14 \times 7$ interconnected $\mu$ disks. The large area LED is of the geometry of a 640 $\times 640 \mu \mathrm{m}^{2}$. The effective emissive areas of the large area, $\mu$ disk and $\mu$ hexagon LEDs are $4.1 \times 10^{5} \mu \mathrm{m}^{2}, \quad 3.7$ $\times 10^{5} \mu \mathrm{m}^{2}$, and $4.2 \times 10^{5} \mu \mathrm{m}^{2}$, respectively, while their respective sidewall circumferences are $2.88 \mathrm{~mm}, 18.03 \mathrm{~mm}$, and $21.77 \mathrm{~mm}$. Subsequently, photolithography was carried out again to define the p-pad and p-pad areas. A bi-layer of Ti/Al (50nm/350nm) was deposited as bonding pads, annealed in $\mathrm{N}_{2}$ at $500{ }^{\circ} \mathrm{C}$ for $1 \mathrm{~min}$. The chips were diced by UV nanosecond laser micromachining and packaged into TO headers. Following Al wire-bonding, a silicone encapsulant was applied to protect the device. The optical spectral were measured with an Ocean Optics HR2000 fiber-coupled spectrometer with optical resolution of $\sim 1 \mathrm{~nm}$. The electrical properties, including the I-V characteristics and leakage cur- 


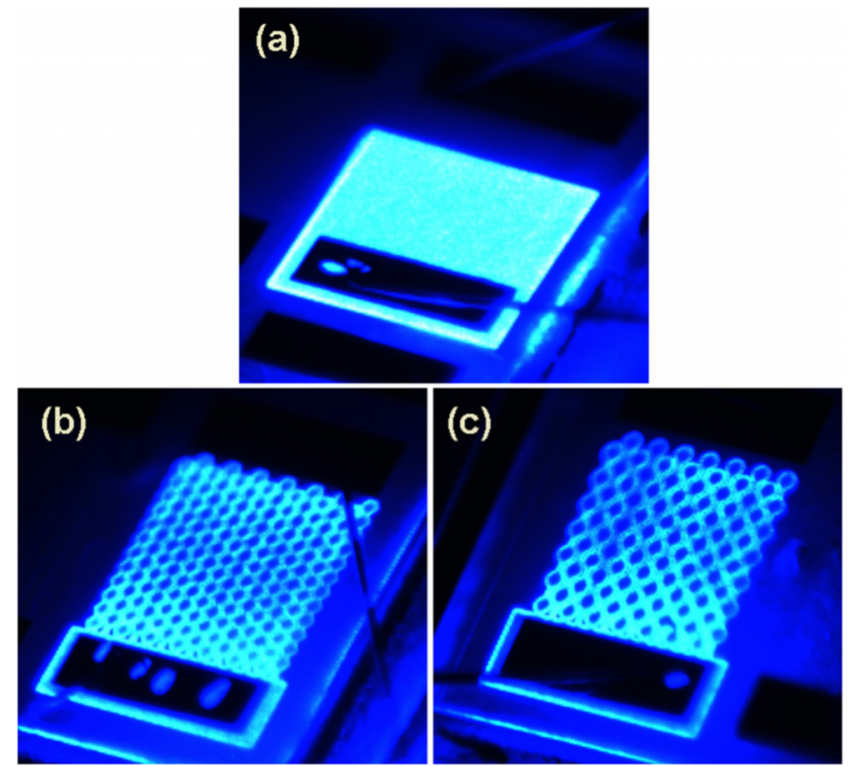

FIG. 1. (Color online) Electroluminescent LEDs of different geometries: (a) large-area; (b) $\mu$ hexagon array; and (c) $\mu$ disk array.

rent of the packaged devices were measured with an HP 4156A precision semiconductor parameter analyzer, while the 1/f noise spectrum was measured by a BTA 9603 FET Noise Analyzer. The devices as shown in Fig. 1, biased at 20 $\mathrm{mA}$, were subsequently subjected to a $900 \mathrm{~h}$ continuous stress test at room temperature.

\section{RESULTS AND DISCUSSION}

Microstructuring of the emission region affects not only the extraction efficiency but also the angular emission characteristics, as a greater component of light is emitted from the sidewalls. As such, the packaged LEDs were placed within a 2 in. integrating sphere to ensure that all the emitted light is collected, while the optical signal was channeled from the output port via fiber to the spectrometer. Figure 2 shows the electroluminescent spectral measured from the devices biased at $20 \mathrm{~mA}$. As noted from the plot, the $\mu \mathrm{LEDs}$ emit $17 \%$ more light than the large-area counterpart, attrib-

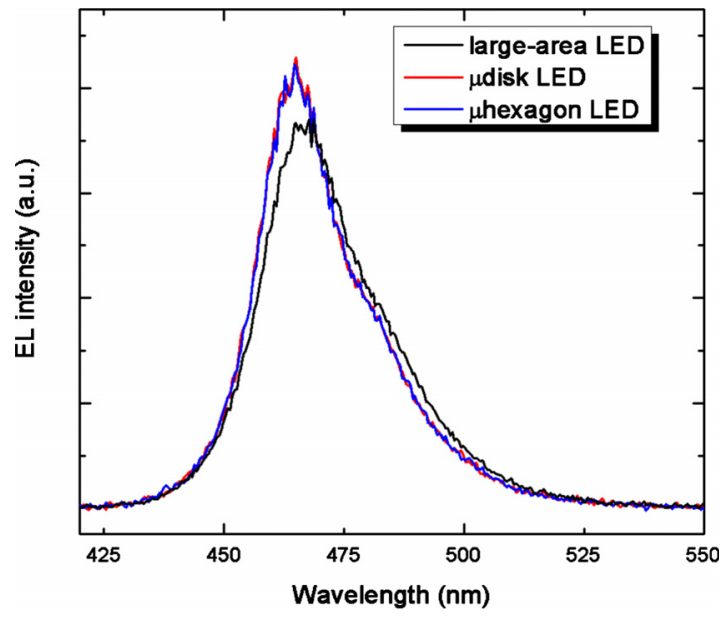

FIG. 2. (Color online) Electroluminescent spectra of the large-area, $\mu$ hexagon, and $\mu$ disk LEDs biased at a current of $20 \mathrm{~mA}$.

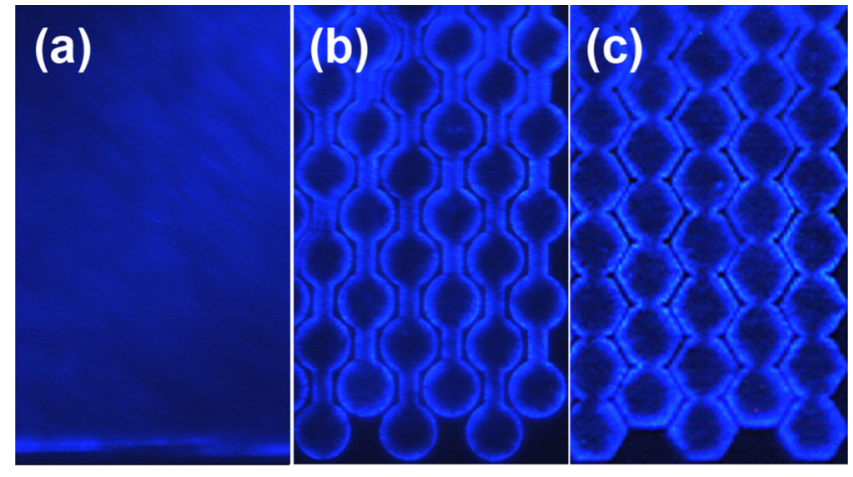

FIG. 3. (Color online) High magnification optical microphotographs showing emission regions of the (a) large-area, (b) $\mu$ disk, and (c) $\mu$ hexagon LEDs.

utable to enhanced light extraction from sidewalls of microstructures. Figures 3(a)-3(c) show high-magnification optical microphotographs of the LEDs biased at lower currents to avoid saturation of the charge-coupled device (CCD) camera. Bright edges were observed at the periphery of the microstructures in Figs. 3(b) and 3(c), contributed by laterally propagating photons that have escaped through the sidewalls. These photons contributed to the overall enhancement of light emission. Additionally, a slight spectral blueshift was observed from the $\mu$ LEDs, which can be attributed to reduced current densities as a result of improved current spreading.

Insight on device reliability is often provided by its electrical characteristics. The current-voltage (I-V) characteristics of the devices are shown in Fig. 4. The leakage currents of the devices have been extracted from the plots at a reverse bias voltage of $4 \mathrm{~V}$. The magnitude of the leakage current increases from $0.79 \mathrm{~mA}$ in large area LED to $3.64 \mathrm{~mA}$ in the $\mu$ hexagon LED and $3.73 \mathrm{~mA}$ in the $\mu$ disk LED. The slope of the linear region of the I-V curves (in the forward bias region) has also been interpolated to determine the static resistance of the devices. The extracted static resistances are

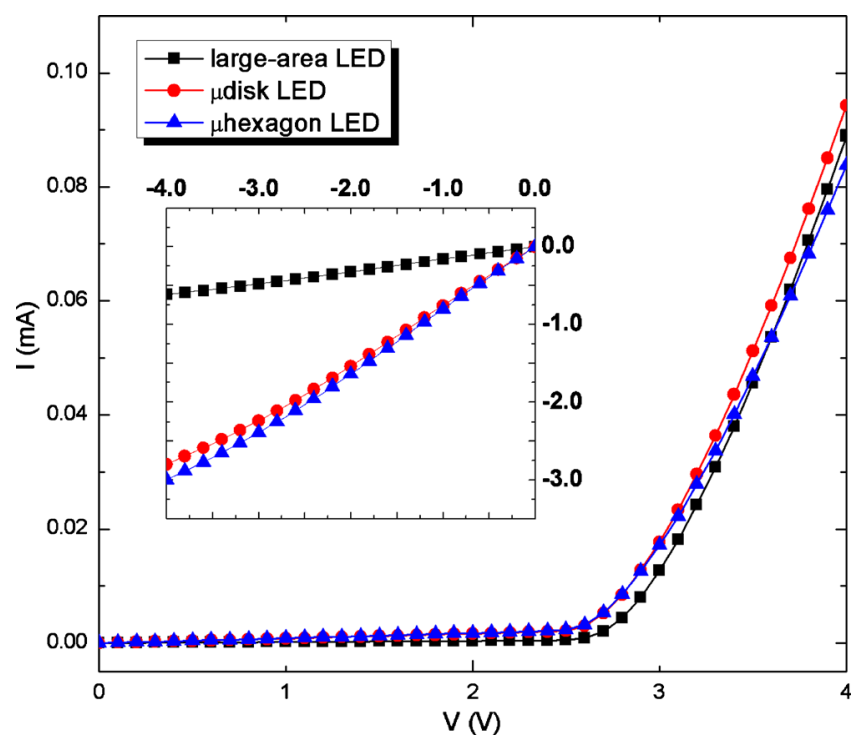

FIG. 4. (Color online) I-V characteristics of the large-area, $\mu$ hexagon, and $\mu$ disk LEDs. 


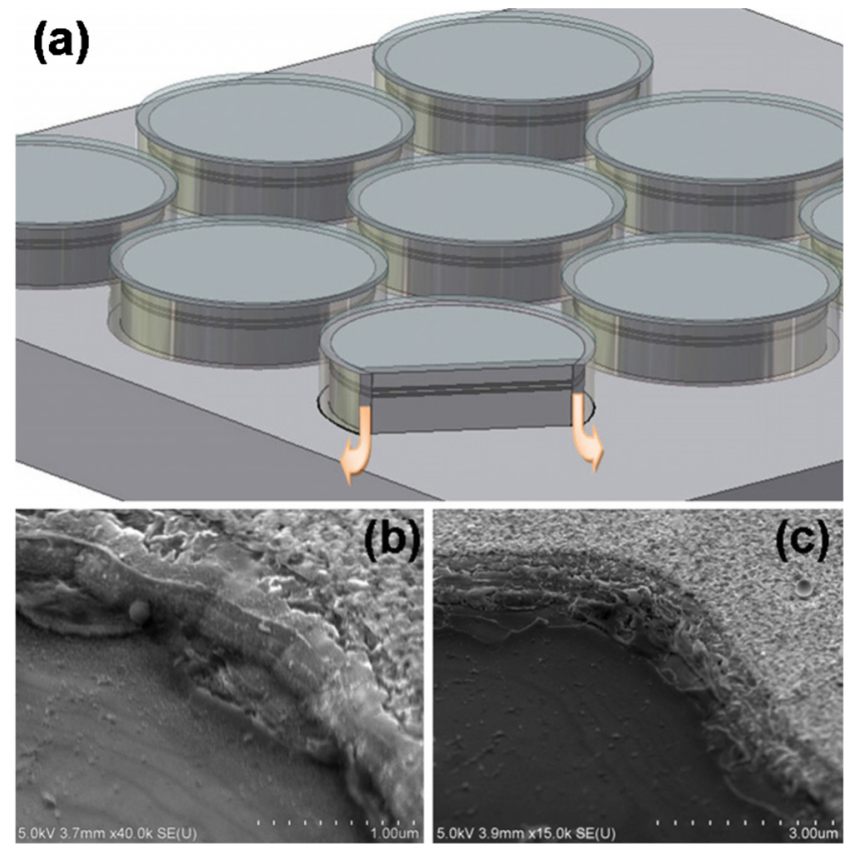

FIG. 5. (Color online) (a) Schematic depicting vertical current flow along sidewalls of $\mu$ LEDs. Close-up SEM images showing sidewalls of (b) asetched and (c) stressed devices.

$13.19 \Omega, 13.69 \Omega$, and $13.64 \Omega$ for the large area, $\mu$ hexagon, and $\mu$ disk LEDs, respectively. From these values, it can be seen that microstructuring has a greater effect on current leakage than on forward conduction. As the emission area shrinks, surface effects become more prominent on the electrical properties. In a $\mu$ LED structure, the MQW active layers are disrupted at the peripheries of individual $\mu \mathrm{LED}$ elements. The exposed sidewall surfaces are highly defective due to the exposure of dangling bonds during plasma etching, whereby the incidence of high energy ions leaves behind crystal damage in the surface regions. ${ }^{5}$ Such defects trap carriers and induces carrier scattering, resulting in a higher static resistance and reduced carrier mobility. ${ }^{6}$

Additionally, these interfacial defects may also serve as vertical current conduction pathways in a way similar to that of threading dislocations. During plasma etching, p-type $\mathrm{GaN}$ becomes compensated through introduction of shallow donors states, ${ }^{7}$ while n-type $\mathrm{GaN}$ becomes $\mathrm{n}+,{ }^{6}$ both of which can be attributed to the formation of nitrogen vacancies as a result of preferential loss of $\mathrm{N}_{2} .{ }^{8}$ Consequently, the $\mu$ LED element can be thought of as being surrounded by a network of defects that are n-type in nature, promoting the vertical conduction of electrons across the $\mathrm{p}-\mathrm{n}$ junction as illustrated in Fig. 5(a), giving rise to an increase in leakage currents. Vertical conductivity along dislocations had been identified as one of the major causes of breakdown in LEDs, ${ }^{9}$ and in p-n junctions in general. In a separate study, the I-V characteristics and lifetimes of a group of LEDs with different dislocation densities (by varying the In concentration in the MQWs) was studied and analyzed, the results of which are summarized in Fig. 6. It was observed that as the dislocation densities of devices increases beyond $\sim 1.5$ $\times 10^{9} \mathrm{~cm}^{-2}$, the leakage currents (at a reverse bias voltage of

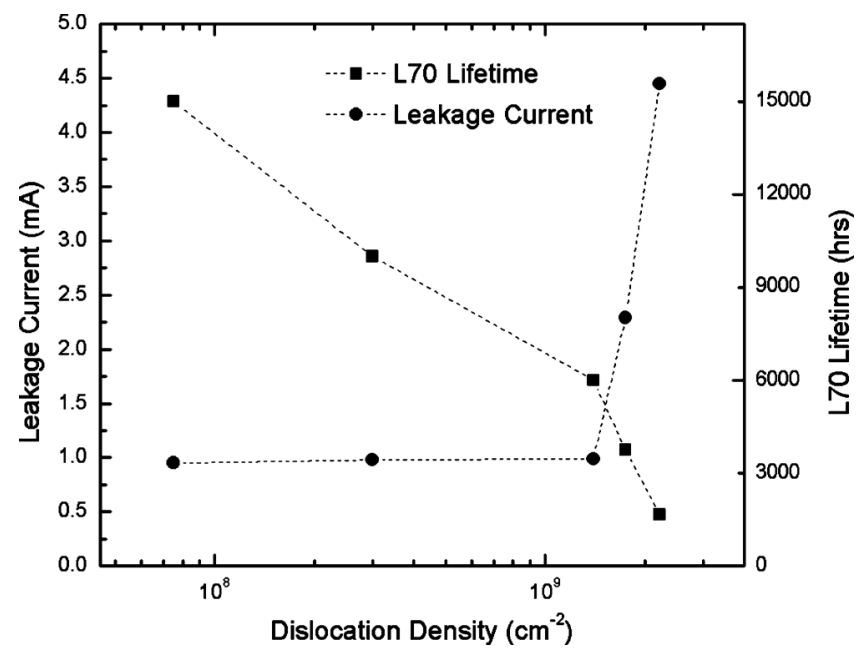

FIG. 6. Plot of reverse leakage currents (at $-4 \mathrm{~V}$ ) and L70 lifetimes as a function of dislocation densities of LEDs.

$4 \mathrm{~V})$ rises rapidly. On the other hand, the L70 lifetime of devices [L70 lifetime is the time to $70 \%$ lumen maintenance, as proposed by ASSIST (Ref. 10)] are drops linearly with increasing dislocation densities. The results positively identify current leakage at sites of dislocations as causes of degradation. Following the same model, the defects generated as a result of microstructuring in $\mu$ LEDs are expected to play a key role in their enhanced degradation rates.

Another possible contributing factor is the junction curvature effect. ${ }^{11}$ The breakdown voltage associated with a spherical junction is significantly reduced compared to a planar junction due to higher field intensities, depending also on the radius of the curvature. The presence of interfacial defects in $\mu$ LEDs is thus predicted to adversely affect their reliability. The SEM images in Fig. 5(b), showing an asetched $\mu$ LED sidewall, and Fig. 5(c), showing the same sidewall after $1000 \mathrm{~h}$ operation, further illustrate this point. The sidewall has become significantly rougher with fragmentation due to proliferation of defects. Low-frequency noise measurements were conducted to further study the carrier properties.

Low-frequency noise, especially $1 / f$ noise and generation-recombination (G-R) noise, is sensitively dependent on the presence of defects, traps and dislocations in semiconductors. The noise spectrum accurately reflects the internal material quality and is thus deemed an important tool for determining the reliability of an LED. ${ }^{12}$ Figure 7 show plots of the noise spectrum of LEDs with different geometries for frequencies in the range of $3.15 \mathrm{~Hz}$ to 16.5 kHz. In an LED (or p-n junction in general) structure, the current spectral density $S_{I}$ under low current bias can be expressed by the following equation: ${ }^{13}$

$$
S_{I}=\frac{4 N_{t} q I_{D}}{\tau_{r} \sigma \nu N_{c}^{2}} e^{2 E_{t} / k T},
$$

where $N_{t}$ is the trap density; a larger $N_{t}$ generally implies poorer crystal quality, increasing the magnitude of the noise spectrum. $\tau_{r}$ is the recombination time or carrier lifetime, which increases the magnitude of the noise spectrum when the trap density increases, while the other terms have their 


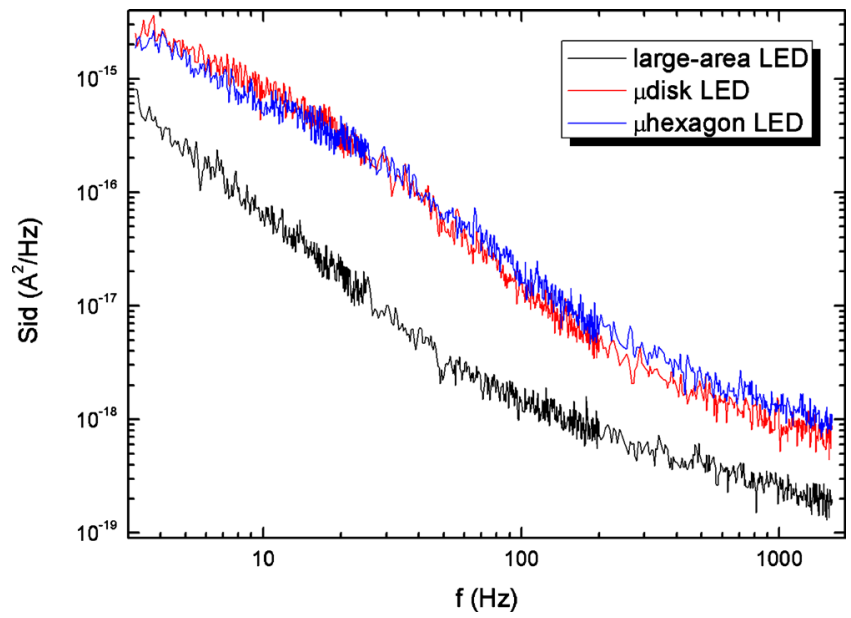

FIG. 7. (Color online) Noise spectrum of LEDs with different geometries.

usual meanings. From Fig. 7, the noise magnitude for a $\mu \mathrm{LED}$ (irrespective of geometry) is approximately an order of magnitude higher than the corresponding large-area LED. This can be understood from the trapping and de-trapping of carriers along the plasma-damaged sidewalls, whereby the total sidewall areas of the $\mu$ LEDs were approximately six to eight times larger than the large-area LED. With a higher sidewall ratio, the noise magnitudes of the LEDs are thus significantly enhanced.

The LEDs were then operated for $>1000$ hrs to determine their rate of degradation. Figure 8 shows the plot of normalized optical intensity over $1000 \mathrm{~h}$, whereby a constant bias current of $20 \mathrm{~mA}$ was applied to the devices. While the two large area LEDs maintains $\sim 82 \%$ and $\sim 79.5 \%$ of their initial optical powers after $900 \mathrm{~h}$, the $\mu$ disk LED maintains $\sim 65.5 \%$ of its initial optical power, while the $\mu$ hexagon LED maintains $\sim 67 \%$ of its initial optical power. During the 900-h test, the optical output of $\mu$ LED degraded very quickly during the first $500 \mathrm{~h}$; the degradation rate slows down since then. On the other hand, the large-area LED degraded almost linearly throughout. Assuming linearity, the L70 lifetime for the large-area LED is $2500 \mathrm{~h}$. For the $\mu$ LEDs, the degradation rate after the initial $100 \mathrm{~h}$ of operation becomes linear, and the lifetime $\mathrm{t}_{70 \%}$ of the $\mu$ disk LED and $\mu$ hexagon LED are found to be $\sim 400 \mathrm{~h}$ and $\sim 500 \mathrm{~h}$, respectively. This corresponds to less than $1 / 5$ of the lifetime of a large-area LED. $\mu$ LEDs, therefore, expected to have shorter lifetimes despite higher extraction efficiency. Expectedly, there is a tradeoff between device performance and reliability. The compromised reliability may be partially alleviated through passivation of sidewalls, control of plasma damage and damage removal, subjects of our on-going studies.

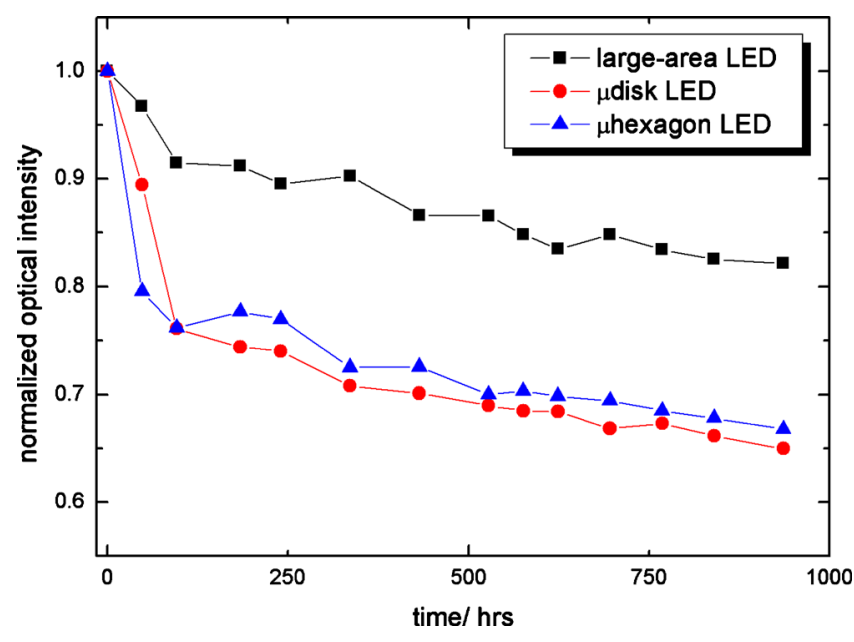

FIG. 8. (Color online) Plot of normalized optical intensity of the large-area, $\mu$ hexagon, and $\mu$ disk LEDs over $1000 \mathrm{~h}$.

\section{CONCLUSION}

In summary, the effects of microstructuring of LEDs have been studied. In terms of optical performance, the increased sidewall surface areas of micro-LEDs certainly promote light extraction efficiency. However, the formation of plasma-damaged sidewalls becomes a threat to the reliability as a result of severe vertical current leakage. A balance should be struck between light extraction and device lifetime.

\section{ACKNOWLEDGMENTS}

This work was supported by a GRF grant of the Research Grant Council of Hong Kong (Project No. HKU 7118/ 09E).

${ }^{1}$ S. X. Jin, J. Li, J. Y. Lin, and H. X. Jiang, Appl. Phys. Lett. 77, 3236 (2000).

${ }^{2}$ H. W. Choi, C. W. Jeon, M. D. Dawson, P. R. Edwards, R. W. Martin, and S. Tripathy, J. Appl. Phys. 93, 5978 (2003).

${ }^{3}$ H. W. Choi, C. W. Jeon, and M. D. Dawson, IEEE Photonics Technol. Lett. 16, 33 (2004).

${ }^{4}$ H. W. Choi and S. J. Chua, J. Vac. Sci. Technol. B 24, 800 (2006).

${ }^{5}$ H. W. Choi, S. J. Chua, A. Raman, J. S. Pan, and A. T. S. Wee, Appl. Phys. Lett. 77, 1795 (2000).

${ }^{6}$ S. J. Chua, H. W. Choi, J. Zhang, and P. Li, Phys. Rev. B 64, 205302 (2001).

${ }^{7}$ X. A. Cao, S. J. Pearton, A. P. Zhang, G. T. Dang, F. Ren, R. J. Shul, L. Zhang, R. Hickman, and J. M. Van Hove, Appl. Phys. Lett. 75, 2569 (1999).

${ }^{8}$ C. R. Eddy, Jr. and B. Molnar, J. Electron. Mater. 28, 314 (1999).

${ }^{9}$ B. S. Simpkins, E. T. Yu, P. Waltereit, and J. S. Speck, J. Appl. Phys. 94, 1448 (2003).

${ }^{10}$ Alliance for Solid-state Illumination and Technologies; website: www.lrc.rpi.edu/programs/solidstate

${ }^{11}$ S. M. Sze and G. Gibbons, Solid-State Electron. 9, 831 (1966).

${ }^{12}$ F. N. Hooge, T. G. M. Kleinpenning, and L. K. J. Vandamme, Rep. Prog. Phys. 44, 479 (1981).

${ }^{13}$ S. Sawyer, S. L. Rumyantsev, M. S. Shur, N. Pala, Yu. Bilenko, J. P. Zhang, X. Hu, A. Lunev, J. Deng, and R. Gaska, J. Appl. Phys. 100, 034504 (2006). 